\title{
Molecular and cellular effects of intraarticular injection of betamethasone in experimental osteoarthritis
}

\author{
M.A. Kabalyk, V.A. Nevzorova, V.S. Dubov, M.A. Tsygankov, T.S. Kovalenko
}

Pacific State Medical University, Vladivostok, Russian Federation

\begin{abstract}
The objective of the study was to assess articular cartilage (AC) and subchondral bone (SCB) remodeling, expression of matrix metalloproteinase 9 (MMP-9) in tissues, vascular endothelial growth factor (VEGF) in experimental osteoarthritis (OA). Material and methods Experimental comparative study was conducted on 12 outbred guinea pigs of both sexes aged 28-30 weeks that were divided into 2 groups of 6 animals each. An injury to the knee joints of hind limbs of control and experimental animals was mechanically simulated by closed scarification using a sterile needle. No treatment was provided for controls. Experimental animals were given an intraarticular injection of betamethasone (BMZ) of $0.1 \mathrm{mg} / \mathrm{kg}$ every two weeks after two weeks of injury. Two subjects of each group were euthanized at 30, 45, 60 days and knee samples collected. Immunohistochemical expression of VEGF and MMP-9 was determined in tissues. Results A statistically significant decrease in VEGF positive chondrocytes and precipitate density, an increase in positive chondrocytes and intensity of tissue response to MMP-9 as compared to those in controls was observed in BMZ animals at 30 days of experiment. A statistically significant decrease in VEGF positive cells and precipitate density, an increase in VEGF positive chondrocytes as compared to those in controls were observed in BMZ animals at 45 days of experiment. Significant reduction in VEGF positive cells and deposit density, significantly higher density of MMP-9 positive precipitates as compared to those in controls were noted at 60 days of BMZ injections. Conclusion Intraarticular injections of BMZ demonstrated a negative effect on AC and SCB with articular tissue remodeling initiated through activation mechanisms of extracellular matrix degradation, as evidenced by high expression of MMP-9. BMZ was shown to block pathological angiogenesis via VEGF inhibition.

Keywords: osteoarthritis, vascular endothelial growth factor, matrix metalloproteinase 9, animal model, articular cartilage, subchondral bone
\end{abstract}

Development and introduction of effective, safe and pathogenetically substantiated therapies for osteoarthritis (OA) is regarded as a priority for the current orthopaedic practice. Degenerative diseases of the musculoskeletal system is an important issue to explore due to high prevalence and incidence of the conditions among the population, working age, in particular, and absence of reliable and effective therapeutic strategies [1]. Non-operative treatments of OA outlined in Osteoarthritis Research Society International (OARSI) guidelines include nonpharmacological and pharmacological interventions employing non-steroidal anti-inflammatory drugs (NSAIDs), acetaminophen, agents with structuremodifying effects, intra-articualr injections of hyaluronic acid and glucocorticosteroids [2]. The clinical use of the latter remains controversial. First, it is suggested that local steroids can be applicable only in presence of synovitis of the OA joint [3]. Second, corticosteriod administration is associated with adverse effect on the biomechanical properties of articular tissues, the articular cartilage, in particular [4]. Third, systemic and local application of glucocorticosteroids has long been known to adversely affect bone quality and develop osteoporosis [5]. Recent studies report clinically relevant short-term benefits of corticosteriod therapy for pain and physical function in OA [2]. There appears to be scant information related to the effects of corticosteriod therapy on the AC and subchondral bone (SCB) despite many-year experience of intra- and peri-articular injections used for OA. There's concern that repeated cortisone injections might damage the cartilage within a joint.

The objective of the study was to assess articular cartilage (AC) and subchondral bone (SCB) remodeling, expression of matrix metalloproteinase 9 (MMP-9) in tissues, vascular endothelial growth factor (VEGF) in experimental OA.

Kabalyk M.A., Nevzorova V.A., Dubov V.S., Tsygankov M.A., Kovalenko T.S. Molecular and cellular effects of intraarticular injection of betamethasone in experimental osteoarthritis. Genij Ortopedii, 2020, vol. 26, no 1, pp. 65-71. DOI 10.18019/10284427-2020-26-1-65-71. (In Russian) 


\section{MATERIAL AND METHODS}

Experimental comparative study was conducted on 12 outbred guinea pigs of both sexes aged 2830 weeks weighing $490-700 \mathrm{~g}$; the animals were housed in standard vivarium cages at the Somov Research Institute of Epidemiology and Immunology. Experiment was carried out in compliance with European Convention for the Protection of Vertebrate Animals used for Experimental and Other Scientific Purposes (adopted by the Council of Europe in 1986), order of the Ministry of Health of the Russian Federation dtd 19.06.2003 № 267 on Approval of the rules of good laboratory practice, in accordance with humane attitudes towards animals stated in the Declaration of Helsinki in 2000 and was approved by multidisciplinary Ethics Committee of FGBOU VO TGMU Minzdrava Rossii.

The animals were divided into 2 groups of 6 animals each. An injury to the knee joints of hind limbs of control $(n=6)$ and experimental $(n=6)$ animals was mechanically simulated by closed scarification using a sterile needle. No treatment was provided for controls. Experimental animals (guinea pigs) were given an intraarticular injection of betamethasone (BMZ/Diprospan, MSD, U.S.A.) of $0.1 \mathrm{mg} / \mathrm{kg}$ every two weeks after two weeks of injury. All invasive manipulations including scarification were performed under general anesthesia using xylazine (Rometar, Bioveta, Czech Republic).Two subjects of each group were euthanized at 30, 45, 60 days using overdose of xylazine and knee samples with simulated injury collected. Specimen were fixed in $10 \%$ neutral buffered formalin during 24 hours, decalcified in electrolyte solution (Biovitrum, Russia) and embedded in paraffin using the standard method. Paraffin sections of $5 \mathrm{mcm}$ were stained with hematoxylin and eosin. Ten histological preparations were obtained from each animal and examined in at least 6 fields of view. Preparations were evaluated with CX41 microscope (Olympus, Japan) equipped with digital camera. Description of micropreparations was performed according to standard protocol. Expression of vascular endothelial growth factor (VEGF) and matrix metalloproteinase 9 (MMP-9) was determined with immunohistochemical staining methods using peroxidase reaction of standard protocol. Primary monoclonal antibodies anti-VEGF-A (Abcam, ab38909, U.S.A.), anti-MMP-9 (Spring, E3660, U.S.A.) avid to proteins of guinea pigs were used in the study. Quantification of immunohistochemical (IHC) image was performed by measuring the precipitate density and \% of positive cells of histochemical reaction, and morphometry produced with ImageJ 4.1 software.

Statistical data analysis was performed using Statistica 10.0 computer program (StatSoft, U.S.A.). Data were expressed as the median (Me) and quartiles [Q25; Q75]. Non-parametric Mann-Whitney test and Wilcoxon rank-sum tests were used for variables that were not normally distributed. For calculations, a significance level of $<0.05$ was adopted.

RESULTS

Ultrastructural organization of the articular tissues was evaluated throughout the experiment (Fig. 1). Cambial chondrocytes appeared to be scattered in the superficial AC a month after closed scarification of the joint of control animals (Fig. 1a). Signs of lost spatial hierarchy were observed in chondrocytes of intermediate layer forming extensive chaotic cells free spaces. Empty chondrocytic lacoons and ectopic reticuloendothelial components could be visualized in the basal AC layer. SCB plate contained neurovascular components in the AC, singular osteons with signs of osteogenesis imperfecta, split bone stock with no signs of reparation. Articular tissues of controls showed slightly narrowed AC and SCB plate after 45-day experiment (Fig. 1b). Erosion with exfoliated cambial layer was noted in the superficial
AC layer. Intermediate and basal zones of the AC contained extensive cell free spaces, differently maturated chondrocytes. Signs of neovascularization and nonadaptive periphearal ossification could be seen in the basal AC. Thinned AC of control animals was characterized by multiple erosion reaching to the intermediate zone at the end of experiment (60 days) (Fig. 1c). Eosinophilic reaction of pericellular matrix was observed in chatiocally scattered chondrocytes. Decrease in SCB thickness in control animals was accompanied by osteo-like matrix and multidirectional connective fibers replacing bone stock at 60 days of experiment.

Superficial erosion and defects of the basal AC were noted in experimental animals that were given an intraarticular injection of BMZ at 30 days 
after two weeks of injury (Fig. 1d). Chondrocytes retained hierarchy of column arrangement but were characterized by basophilic reaction of pericellular matrix. Singular ossification components could be seen in the lower cartilaginous zones. Multiple heterogenous components of neuroangiointegration were observed in the SCB plate with destruction of bone stock showing no signs of reparation. Superficial erosion of the cartilage was observed with BMZ injection at 45 days of experiment (Fig. 1e) in presence of extensive cell free spaces. No signs of hierarchy could be seen in the spatial arrangement of hypertrophied chondrocytes and colorless cytoplasm and basophilic pericellular matrix. The basal AC zone having no osteochondral line appeared to merge with basophilic substance of the SCB plate characterized by multiple erosion with no signs of reparation. Considerable thinned AC and SCB could be visualized at the end of experiment (Fig. 1f). Signs of hypertrophic differentiation were representative of chondrocytes with clusters disarranged. SCB was presented with osteo-like matrix and singular active osteoclasts in cavities of resorption.
Analysis of VEGF (Fig. 2) and MMP-9 (Fig. 3) expression in articular tissues of BMZ animals showed (Table) statistically significant decrease in VEGF positive chondrocytes and precipitate density $(\mathrm{z}=-2.3, \mathrm{p}=0.02 ; \mathrm{z}=-2.2, \mathrm{p}=0.03$, respectively), an increase in VEGF positive chondrocytes and intensity of tissue response to MMP-9 $(\mathrm{z}=3.3$, $p=0.0009 ; z=2.6, p=0.01$, respectively) compared to those in controls observed at 30 days of experiment. A statistically significant decrease in VEGF positive cells and precipitate density $(\mathrm{z}=-2.6, \mathrm{p}=0.01$; $z=-3.3, p=0.0009$, respectively), an increase in VEGF positive chondrocytes $(z=3.3, p=0.0009)$ compared to those in controls were observed in BMZ animals at 45 days of experiment. Significant reduction in VEGF positive cells and deposit density $(z=-2.9, p=0.004 ; z=-3.3, p=0.0009)$, significantly higher density of MMP-9 positive precipitates $(z=2.5, p=0.01)$ as compared to those in controls were noted in BMZ animals at 60 days of experiment. There was no statistically significant differences in MMP-9 positive chondrocytes as compared to those in controls $(\mathrm{z}=0.8, \mathrm{p}=0.4)$.

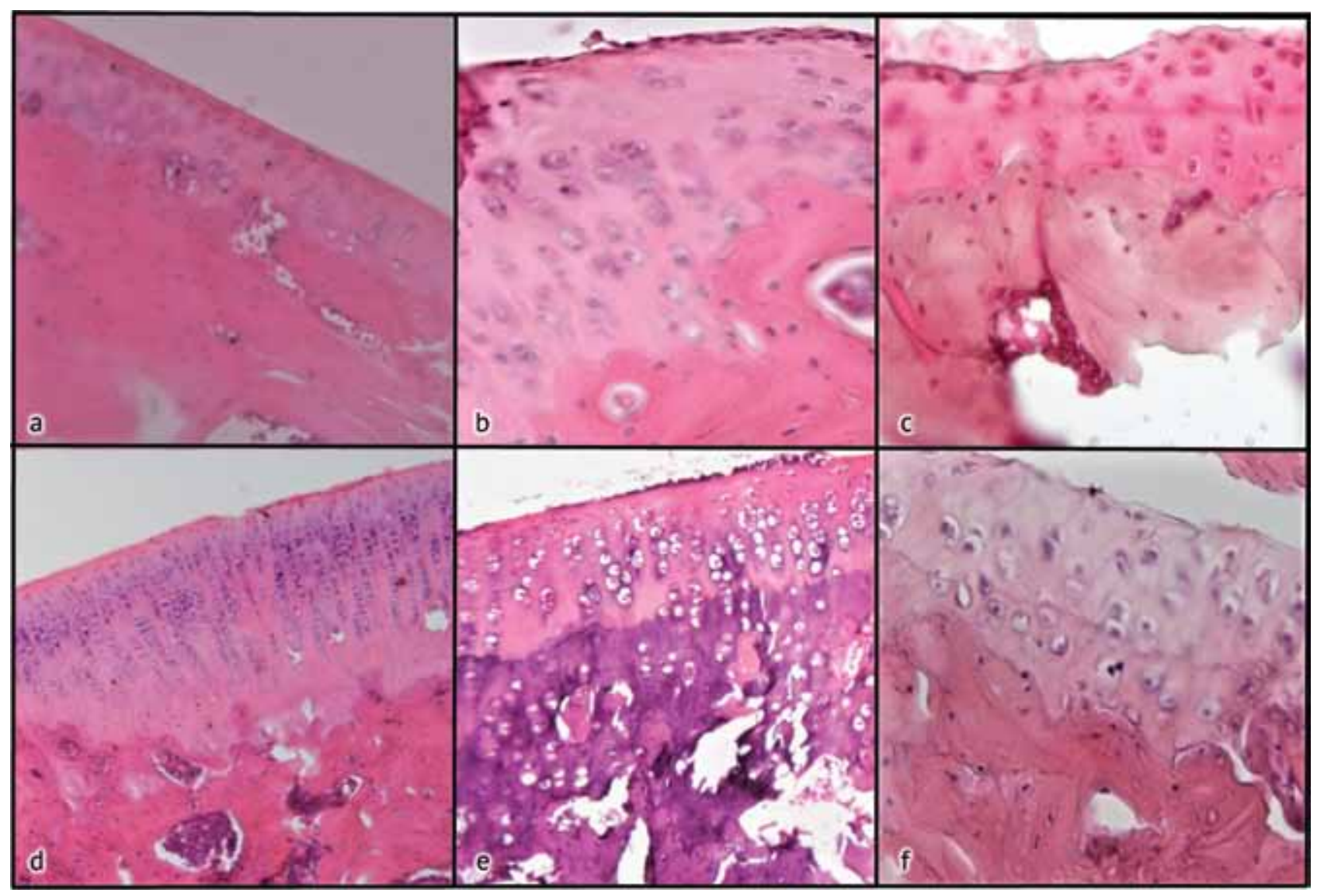

Fig. 1 Representative images of the AC and SCB obtained during experiment in controls $(\boldsymbol{a}, \boldsymbol{b}, \boldsymbol{c})$ and experimental animals that received intraarticular injection of BMZ $(\boldsymbol{d}, \boldsymbol{e}, \boldsymbol{f})$. Images taken at 30 days $(\boldsymbol{a}, \boldsymbol{d}), 45$ days $(\boldsymbol{b}, \boldsymbol{e})$ and 60 days $(\boldsymbol{c}, \boldsymbol{f})$ of experiment. Stained with $H \& E$, magnification $\times 200$. Description provided in the text 


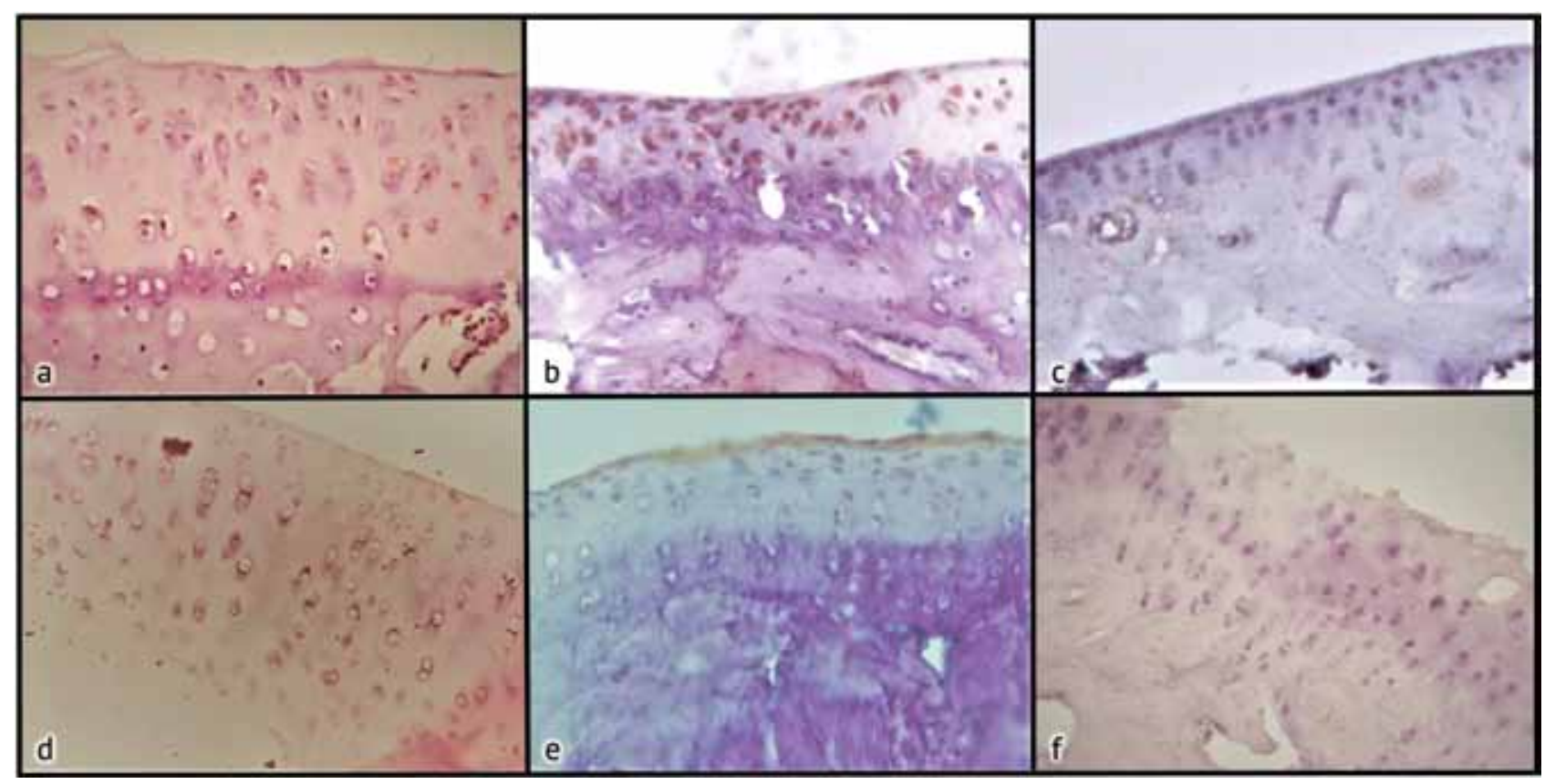

Fig. 2 Representative images of VEGF expression in the AC and SCB during experiment in controls $(\boldsymbol{a}, \boldsymbol{b}, \boldsymbol{c})$ and experimental animals that received intra-articular injection of BMZ $(\boldsymbol{d}, \boldsymbol{e}, \boldsymbol{f})$. Images taken at 30 days $(\boldsymbol{a}, \boldsymbol{d}), 45$ days $(\boldsymbol{b}, \boldsymbol{e})$ and 60 days $(\boldsymbol{c}, \boldsymbol{f})$ of experiment. Immunohistochemistry, magnification $\times 200$. Description provided in the text

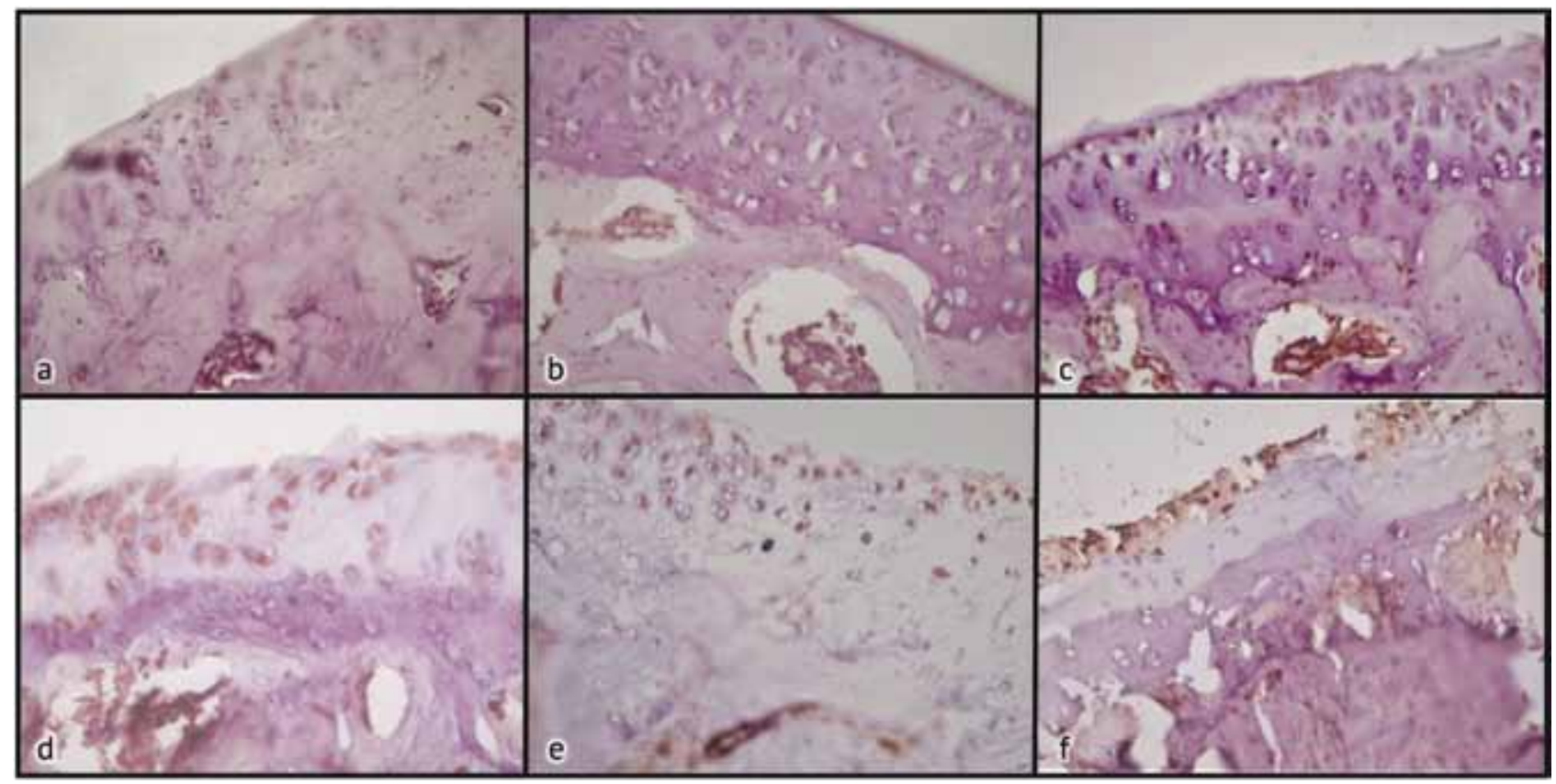

Fig. 3 Representative images of MMP-9 expression in the AC and SCB during experiment in controls $(\boldsymbol{a}, \boldsymbol{b}, \boldsymbol{c})$ and experimental animals that received intra-articular injection of BMZ $(\boldsymbol{d}, \boldsymbol{e}, \boldsymbol{f})$. Images taken at 30 days $(\boldsymbol{a}, \boldsymbol{d}), 45$ days $(\boldsymbol{b}, \boldsymbol{e})$ and 60 days $(\boldsymbol{c}, \boldsymbol{f})$ of experiment. Immunohistochemistry, magnification $\times 200$. Description provided in the text

Dynamics in VEGF and MMP-9 expression was evaluated in the articular tissues during experiment. Statistically significant increase in VEGF and MMP9-positive chondrocytes, VEGF precipitate density $(\mathrm{w}=2.5, \mathrm{p}=0.02 ; \mathrm{w}=2.6, \mathrm{p}=0.009 ; \mathrm{w}=2.5$, $\mathrm{p}=0.01$, respectively) except for MMP-9 precipitate density ( $\mathrm{w}=1.1, \mathrm{p}=0.3$ ) was observed in controls at 45 days of experiment. Statistically significant increase in MMP-9-positive chondrocytes, VEGF and MMP-9 precipitate density $(\mathrm{w}=2.3, \mathrm{p}=0.03$; $\mathrm{w}=2.9, \mathrm{p}=0.004 ; \mathrm{w}=2.6, \mathrm{p}=0.009)$ except for VEGF-positive AC cells $(\mathrm{w}=-1.1, \mathrm{p}=0.01$ ) was noted at the end of experiment. Statistically significant decrease in VEGF positive cells, VEGF and MMP-9 precipitate density $(\mathrm{w}=-2.3, \mathrm{p}=0.03 ; \mathrm{w}=-2.5$, $\mathrm{p}=0.01 ; \mathrm{w}=-2.5, \mathrm{p}=0.01)$ was observed in controls at 45 days of experiment.

Statistically significant decrease in MMP-9 precipitate density ( $\mathrm{w}=-2.1, \mathrm{p}=0.04)$ and increase in VEGF precipitate density $(\mathrm{w}=2.5, \mathrm{p}=0.01$ ) was 
recorded in BMZ animals at 45 days of experiment. There was no statistically significant differences in chondrocytes being positive for VEGF and MMP-9 $(\mathrm{w}=-2.0, \mathrm{p}=0.05 ; \mathrm{w}=1.8, \mathrm{p}=0.07$, respectively $)$ compared to baseline parameters. Statistically significant increase in VEGF and MMP-9 precipitate density $\quad(\mathrm{w}=2.3, \quad \mathrm{p}=0.03 ; \mathrm{w}=2.5, \mathrm{p}=0.01$, respectively) and decrease in MMP-9 positive cells $(\mathrm{w}=-2.5, \mathrm{p}=0.01)$ were noted in BMZ animals at the end of experiment. VEGF precipitate density and chondrocytes positive for MMP- $9(\mathrm{w}=-2.6, \mathrm{p}=0.01$; $\mathrm{w}=2.5, \mathrm{p}=0.01$, respectively) appeared to decrease while MMP-9 precipitate density VEGF $(\mathrm{w}=2.6$, $\mathrm{p}=0.01$ ) increased as compared to measurements at 45 days of experiment. There were no statistically significant differences in VEGF positive chondrocytes at 30 and 45 days of experiment $(\mathrm{w}=-0.6, \mathrm{p}=0.6$; $\mathrm{w}=-1.8, \mathrm{p}=0.07)$.

Table

VEGF and MMP-9 expression in the AC and SCB of study animals (Me [Q25; Q75])

\begin{tabular}{|c|c|c|}
\hline Parameter, unit of measurement & Controls $(n=6)$ & BMZ animals $(n=6)$ \\
\hline \multicolumn{3}{|c|}{30 days } \\
\hline VEGF positive cells, \% & $74.0[71.7 ; 79.3]$ & $61.6[57.6 ; 64.8] \times$ \\
\hline VEGF precipitate density, c.u. & $99.3[95.0 ; 103.3]$ & $76.0[70.4 ; 81.7] \times$ \\
\hline MMP-9 positive cells, \% & $45.6[42.1 ; 48.4]$ & $94.3[91.9 ; 95.8] \times$ \\
\hline MMP-9 precipitate density, c.u. & $81.9[66.6 ; 97.3]$ & $112.6[106.4 ; 123.8] \times$ \\
\hline \multicolumn{3}{|c|}{45 days } \\
\hline VEGF positive cells, \% & $93.2[91.4 ; 94.5]^{*}$ & $54.0[49.3 ; 57.6] \times$ \\
\hline VEGF precipitate density, c.u. & $143.9[143.0 ; 151.6]^{*}$ & $110.8[107.8 ; 112.6] \times *$ \\
\hline MMP-9 positive cells, \% & $56.8[54.0 ; 59.2]^{*}$ & $96.1[94.1 ; 98.1] \times$ \\
\hline MMP-9 precipitate density, c.u. & $99.1[80.3 ; 110.9]$ & $107.5[101.5 ; 112.3] *$ \\
\hline \multicolumn{3}{|c|}{60 days } \\
\hline VEGF positive cells, \% & $79.2[71.0 ; 87.3] \#$ & $56.3[51.3 ; 59.6] \times$ \\
\hline VEGF precipitate density, c.u. & $125.3[123.9 ; 126.8]{ }^{*} \#$ & $91.3[83.6 ; 95.3] \times * \#$ \\
\hline MMP-9 positive cells, \% & $51.8[49.7 ; 56.9] *$ & $53.4[51.5 ; 56.3] * \#$ \\
\hline MMP-9 precipitate density, c.u. & $125.3[123.9 ; 126.8]{ }^{*} \#$ & $135.9[128.3 ; 143.9] \times * \#$ \\
\hline
\end{tabular}

Note: * differences in the group compared to baseline values measured at 30 days of experiment were statistically significant at $\mathrm{p}<0.05$; \# differences in the group compared to the values measured at 45 days of experiment were statistically significant at $\mathrm{p}<0.05$; $x$ differences compared to controls were statistically significant at $\mathrm{p}<0.05$

\section{DISCUSSION}

The pathogenesis of $\mathrm{OA}$ is multifactorial and extremely complex due to a great number of mollecular and cellular mechanisms involved. Essential contributors to articular remodeling have been identified in recent decades including heat shock proteins, matrix metalloproteinases, growth factors and cell differentiation [6]. Pathological articular angioproliferation due to VEGF expression is known to contribute to OA initiation and progression [7]. Elevated VEGF levels were found to be associated with $\mathrm{OA}$ structural progression [8]. While previous studies focused on systemic/ plasma VEGF concentration in OA our research demonstrated local articular VEGF hyperexpression in experimental animals. VEGF concentration in the $\mathrm{AC}$ was found to increase with OA progression and be accompanied by pathological angioproliferation and hypertrophied chondrocytes. This is in line with other series exploring pathogenetic VEGF effect due to the increased number and permeability of vessels [9], capacity to lead to hypertrophy and formation of the osteoblastic phenotype of chondrocytes [10].

MMPs are known to have a direct effect on the extracellular AC matrix facilitating its degradation and replacement with coarse fibrous connective tissues [11]. MMP-9 is thought to be involved in OA through degradation of aggrecan, the major proteoglycan in articular cartilage [12]. The series iudicated to MMP-9 playing an essential role in the structural progression of OA. MMP-9 concentration in tissues was shown to increase over time and be associated with thinned AC and SCB replaced with osteo-like coarse fibrous calcific matrix. The non-adaptive remodeling of the SCB plate inevitably leads to the loss of trophic patterns of the AC and hypoxia [7]. It is expedient to evaluate an effect of the existing pharmacological interventions on specific molecular patterns taking into consideration available findings. 
The study explored the effect of intraarticular injection of BMZ on AC tissues in experimental OA. $\mathrm{BMZ}$ injection was found to facilitate progressive decrease in thickness of the AC and the SCB plate. However, BMZ injection was likely to refrain AC degradation as compared to controls due to more rapid changes in synthetic activity of chondrocytes observed at 30 days of experiment (two weeks after the primary BMZ injection) and appeared as a considerably changed reaction of the pericellular matrix. We can suggest that chondrocytes acquired osteogenic phenotype being characterized by hypertrophy and partical ossification of basal postion of the SCB plate to be substituted by loss of boundaries between the AC and SCB. The assumption is on par with other series reporting deleterious effects of corticosteroids on chondrocytes and cartilage degradation rate [13]. Interestingly, BMZ animals showed no ectopic angiogenesis of the AC revealed in controls. It was associated with lower VEGF expression following BMZ injection as compared to controls that indicated to a role of the factor in pathological angiogenesis of the AC. The phenomenon was likely to be related with the capability of BMZ to block some external stimuli through subunit p65 Nf-kB that activated VEGF [4]. Active degradation of the AC and SCB is accompanied by hyperexpression of MMP-9. Although a role of corticosteroids in activation of MMPs in the AC is poorly understood, potentials of stimulating expression of MMP-9 in the bone are well known [14]. It could serve an explanation to greater changes observed in SCB that progressively got thinner with progression of the disease and BMZ injection. Intraosseous activation of reticuloendothelial components appeared to be substituted with bone destruction and non-adaptive regeneration with interim bone stock being replaced with coarse fibrous (osteo-like) calcific matrix. On the whole, SCB reaction on BMZ goes straight into a well-accepted concept of corticosteroids inducing osteoporosis [5].

Researchers have discovered important details about the pathogenesis of lupus in recent years that could help lead to more effective therapy.

\section{CONCLUSION}

The findings showed that intraarticular injections of BMZ had a deleterious effect on the AC and SCB. Repeat BMZ injections applied once in two weeks was characterized by thinned AC, impaired cellular hierarchy and phenotype of chondrocytes. Impaired osteogenesis and osteoreparation in the SCB finally resulted in thinned SCB plate. Articular tissue remodeling was initiated through activation mechanisms of extracellular matrix degradation, as evidenced by high expression of MMP-9. BMZ was shown to block pathological angiogenesis via VEGF inhibition. Findings on OA pathogenesis provided weak evidence on the potential benefits of intraarticular corticosteroid injections performed to treat OA. Intraarticular injections of the drugs must be restricted in acute phases of synovial inflammation.

Funding. No financial sponsorship was provided for the research which was produced as a part of an independent study project.

Competing interests. The authors have declared that no competing interest exists.

\section{REFERENCE}

1. Kabalyk M.A. Rasprostranennost osteoartrita v Rossii: regionalnye aspekty dinamiki statisticheskikh pokazatelei za 2011-2016 gg. [Osteoarthritis prevalence in Russia: regional aspects of the dynamics of statistical values for 2011-2016]. Nauchno-prakticheskaia Revmatologiia, 2018, vol. 56, no. 4, pp. 416-422. (in Russian) DOI: 10.14412/1995-4484-2018-416-422.

2. McAlindon T.E., Bannuru R.R., Sullivan M.C., Arden N.K., Berenbaum F., Bierma-Zeinstra S.M., Hawker G.A., Henrotin Y., Hunter D.J., Kawaguchi H., Kwoh K., Lohmander S., Rannou F., Roos E.M., Underwood M. OARSI guidelines for the non-surgical management of knee osteoarthritis. Osteoarthritis Cartilage, 2014, vol. 22, no. 3, pp. 363-388. DOI: 10.1016/j.joca.2014.01.003.

3. Frean S.P., Cambridge H., Lees P. Effects of anti-arthritic drugs on proteoglycan synthesis by equine cartilage. J. Vet. Pharmacol. Ther., 2002, vol. 25, no. 4, pp. 289-298.

4. Sun F., Zhang Y., Li Q. Therapeutic mechanisms of ibuprofen, prednisone and betamethasone in osteoarthritis. Mol. Med. Rep., 2017, vol. 15, no. 2, pp. 981-987. DOI: 10.3892/mmr.2016.6068.

5. Milyani A.A., Al-Agha A.E. Implication of Topical Steroids in the Onset of Osteoporosis. Case Rep. Pediatr., 2018, vol. 2018, pp. 4802172. DOI: $10.1155 / 2018 / 4802172$.

6. Kabalyk M.A. Age-related aspects of the involvement of heat shock proteins in the pathogenesis of osteoarthritis. Adv. Gerontol., 2017, vol. 30, no. 3, pp. 341-346. 
7. Kabalyk M.A., Plekhova N.G., Lagureva A.V., Suniaikin A.B. Kliniko-patogeneticheskie vzaimosviazi molekuliarnoi reguliatsii apoptoza i kletochnoi differentsirovki pri osteoartrite [Clinical-and-pathogenetic relationships of regulating apoptosis and cell differentiation in osteoarthritis]. Kazanskii Meditsinskii Zhurnal, 2018, vol. 99, no. 1, pp. 30-36. (in Russian)

8. Yuan Q., Sun L., Li J.J., An C.H. Elevated VEGF levels contribute to the pathogenesis of osteoarthritis. BMC Musculoskelet. Disord., 2014, no. 15, pp. 437. DOI: 10.1186/1471-2474-15-437.

9. Cebi H., Akşahin E., Yüksel H.Y., Celebi L., Aktekin C.N., Hapa O., Muratli H.H., Biçimoğlu A. Plasma vascular endothelial growth factor levels are similar in subjects with and without osteoporosis. Eklem. Hastalik. Cerrahisi., 2010, vol. 21, no. 2, pp. 91-97.

10.Neve A., Cantatore F.P., Corrado A., Gaudio A., Ruggieri S., Ribatti D. In vitro and in vivo angiogenic activity of osteoarthritic and osteoporotic osteoblasts is modulated by VEGF and vitamin D3 treatment. Regul. Pept., 2013, vol. 184, pp. 81-84. DOI: 10.1016/j. regpep.2013.03.014.

11.Ko J.H., Kang Y.M., Yang J.H., Kim J.S., Lee W.J., Kim S.H., Yang I.H., Moon S.H. Regulation of MMP and TIMP expression in synovial fibroblasts from knee osteoarthritis with flexion contracture using adenovirus-mediated relaxin gene therapy. Knee, 2019, vol. 26, no. 2, pp. 317-329. DOI: 10.1016/j.knee.2019.01.010.

12.Lipari L., Gerbino A. Expression of gelatinases (MMP-2, MMP-9) in human articular cartilage. Int. J. Immunopathol. Pharmacol., 2013, vol. 26, no. 3, pp. 817-823.

13.Wernecke C., Braun H.J., Dragoo J.L. The Effect of Intra-articular Corticosteroids on Articular Cartilage: A Systematic Review. Orthop. J. Sports Med., 2015, vol. 3, no. 5, pp. 2325967115581163. DOI: 10.1177/2325967115581163.

14.Sun B., Sun J., Han X., Liu H., Li J., Du J., Feng W., Liu B., Cui J., Guo J., Amizuka N., Li M. Immunolocalization of MMP 2, 9 and 13 in prednisolone induced osteoporosis in mice. Histol. Histopathol., 2016, vol. 31, no. 6, pp. 647-656. DOI: 10.14670/HH-11-702.

Received: 02.07.2019

Сведения об авторах:

Information about the authors:

1.Maksim A. Kabalyk, M.D., Ph.D., Pacific State Medical University, Vladivostok, Russian Federation, Email:maxi_maxim@mail.ru

2. Vera A. Nevzorova, M.D., Ph.D., Professor,

Pacific State Medical University, Vladivostok, Russian Federation,

Email: smu.tgmu@gmail.com

3. Vitaliy S. Dubov,

Pacific State Medical University, Vladivostok, Russian Federation,

Email: afrosamurai777@mail.ru

4. Mikhail A. Tsygankov,

Pacific State Medical University, Vladivostok, Russian Federation,

Email: mk86prim@gmail.com

5. Tatiana S. Kovalenko,

Pacific State Medical University, Vladivostok, Russian Federation,

Email: tanechka.agent005@gmail.com 